\title{
The impact of urine microbiota in patients with lower urinary tract symptoms
}

\author{
Hsiang-Ying Lee 1,2,3,4, Jiunn-Wei Wang ${ }^{5,6}$, Yung-Shun Juan 1,3,4 ${ }^{7}$ Ching-Chia Li $i^{3,4}$, Chung-Jung Liu' ${ }^{6}$ \\ Sung Yong Cho ${ }^{7}$, Hsin-Chih Yeh ${ }^{1,3,4}$, Kuang-Shun Chueh', Wen-Jeng Wu ${ }^{3,4,6^{*}}$ and Deng-Chyang Wu ${ }^{5,6,8^{*}}$
}

\begin{abstract}
Introduction: Inflammation and infection are causative factors of benign prostatic hyperplasia (BPH). Urine is not sterile, and urine microbiota identified by DNA sequencing can play an important role in the development of BPH and can influence the severity of lower urinary tract symptoms (LUTS).

Materials and methods: We collected mid-stream voided urine samples from BPH patients and control participants and stored them in a freezer at $-80^{\circ} \mathrm{C}$. All enrolled participants were requested to provide information about their clinical characteristics and complete the International Prostate Symptom Score (IPSS) questionnaire. Each step of the procedure, including the extraction of the genomic DNA from the urine samples; the amplification by polymerase chain reaction (PCR); PCR product quantification, mixing, and purification; DNA library preparation; and sequencing was performed with quality control (QC) measures. Alpha diversity was indicative of the species complexity within individual urine samples, and beta diversity analysis was used to evaluate the differences among the samples in terms of species complexity. Pearson's correlation analysis was performed to calculate the relationship between the clinical characteristics of the participants and the microbiota species in the urine samples.
\end{abstract}

Results: We enrolled $77 \mathrm{BPH}$ patients and 30 control participants who reported no recent antibiotic usage. Old age, high IPSS and poor quality of life were observed in the participants of the BPH group. No significant differences were observed in the alpha diversity of the samples. In the beta diversity analysis, there was a significant difference between the microbiota in the samples of the BPH and control groups according to ANOSIM statistical analysis. On comparing the groups, the ten bacterial genera present in the samples of the BPH group in descending order of abundance were: Sphingomonas, Bacteroides, Lactobacillus, Streptococcus, Alcaligenes, Prevotella, Ruminococcaceae UCG-014, Escherichia_Shigella, Akkermansia, and Parabacteroides. Spearman's correlation analysis revealed that urine samples showing the presence of the bacterial genera Haemophilus, Staphylococcus, Dolosigranulum, Listeria, Phascolarctobacterium, Enhydrobacter, Bacillus, [Ruminococcus]torques, Faecalibacterium, and Finegoldia correlated with a high IPSS, and severe storage and voiding symptoms $(P<0.05)$.

Conclusion: Our current study shows that dysbiosis of urine microbiota may be related to the development of BPH and the severity of LUTS. Further research targeting specific microbes to identify their role in the development of diseases is necessary and might provide novel diagnostic biomarkers and therapeutic options.

\footnotetext{
*Correspondence: wejewu@kmu.edu.tw; ashum1009@gmail.com

${ }^{3}$ Department of Urology, Kaohsiung Medical University Hospital,

Kaohsiung, Taiwan

${ }^{5}$ Division of Gastroenterology, Department of Internal Medicine,

Kaohsiung Medical University Hospital, Kaohsiung Medical University,

Kaohsiung, Taiwan

Full list of author information is available at the end of the article
}

(c) The Author(s) 2021. This article is licensed under a Creative Commons Attribution 4.0 International License, which permits use, sharing, adaptation, distribution and reproduction in any medium or format, as long as you give appropriate credit to the original author(s) and the source, provide a link to the Creative Commons licence, and indicate if changes were made. The images or other third party material in this article are included in the article's Creative Commons licence, unless indicated otherwise in a credit line to the material. If material is not included in the article's Creative Commons licence and your intended use is not permitted by statutory regulation or exceeds the permitted use, you will need to obtain permission directly from the copyright holder. To view a copy of this licence, visit http://creativeco mmons.org/licenses/by/4.0/. The Creative Commons Public Domain Dedication waiver (http://creativecommons.org/publicdomain/ zero/1.0/) applies to the data made available in this article, unless otherwise stated in a credit line to the data. 
Keywords: Urine microbiota, Benign prostate hyperplasia, Lower urinary tract symptoms

\section{Introduction}

Benign prostatic hyperplasia (BPH) is one of the most common causes contributing to the lower urinary tract symptoms (LUTS) in elderly male patients, and its prevalence increases with age [1]. A previous meta-analysis revealed a prevalence of $\mathrm{BPH}$ in $26.2 \%$ of the participants tested [2]. LUTS can impact the quality of life and disturb routine activities. BPH is defined as the histological proliferation of connective tissue, smooth muscle, and epithelial cells, predominantly within the prostatic transition zone. Inflammation may increase the risk and severity of $\mathrm{BPH}$ and LUTS, although the underlying mechanism is unclear. Metabolic risk factors such as obesity induce inflammatory processes that are associated with the prevalence of BPH [3]. Systemic inflammation and oxidative stress induced by metabolic syndromes may lead to the BPH proliferative pathway [4]. Previous studies have discovered that the severity of the histological inflammatory conditions in the prostate specimen is associated with the extent of prostate enlargement [5]. Additionally, infection has been recognized as a factor related to the increased severity of BPH symptoms, while prior gonorrhea or prostatitis increases the possibility of occurrence of LUTS and the requirement of surgery for BPH $[6,7]$.

Human microbiota, which is the collective term for microorganisms in the human body comprising bacteria, viruses, parasites, and fungi, maintains symbiosis in the body, including the genitourinary tract. This influence of the human microbiota on human health and disease development is verified by emerging evidence. Dysbiosis, which is defined as an alteration in the normal composition of the microbiota, may be associated with diseases [8]. Previously, urine was considered sterile based on the results of the standard clinical culture tests. However, urine is no longer considered sterile, since the advancement of high-throughput DNA sequencing, by which bacteria can be detected in urine samples of even culture-negative healthy individuals [9-11]. Furthermore, alterations in the urine microbiota composition and in the environment were noted in various urologic diseases. By using 16S rRNA gene sequencing, previous studies have shown that the characteristics of urine microbiota are related to an increase in the episodes of urge urinary incontinence (UUI) in adult women [1214]. The results obtained by $16 \mathrm{~S}$ rRNA sequencing were also supported and complemented by enhanced urine culture methods although standard urine culture is negative [15-18]. Pearce et al. demonstrated that in nearly half of the sequence-positive samples in UUI patients,
Lactobacillus and Gardnerella were the most common urotypes [14]. Escherichia coli and Enterococcus showed diversity in distribution between the samples of the patients with prostate cancer and BPH [19]. Therefore, ecological dysbiosis in urine may play an important role in the pathogenesis of prostate diseases [19]. In brief, urine microbiota may impact disease pathogenesis in both genders.

Standard culture-negative urine does not preclude the presence of urinary microbiota, which may still contribute to the development of BPH. To verify this possibility, we compared the composition of the urinary microbiota in $\mathrm{BPH}$ patients to that in non-BPH participants by $16 \mathrm{~S}$ rDNA amplicon sequencing. According to recent Bajic et al. study, severity of LUTS is related to bladder microbiome [20]. In addition, we also investigated the relationship between the bacterial population in the urine samples and the clinical parameters in the participants, including the severity of LUTS, quantified using a questionnaire in the present study.

\section{Materials and methods}

\section{Patient population and urine collection}

All procedures involving human participants were reviewed and approved by the Kaohsiung Medical University Hospital Institutional Review Board (KMUHIRBF(I)-20,190,013). Informed consent was obtained from all participants, and all experiments were performed in accordance with relevant guidelines and regulations. We recruited a cohort of patients with $\mathrm{BPH}$, undergoing medical treatment for LUTS. Participants with other diseases that could interfere with voiding conditions, such as urinary tract infection, abnormal urinary tract anatomy, hereditary and congenital diseases, neurological diseases, malignancy, and history of spinal injury were excluded. Males without LUTS were enrolled as participants of the control group. Participants were also excluded if they had received an antibiotic treatment in the past two months, or a recent indwelling catheter. For each participant, mid-stream voided urine was collected with a sterile tube and stored in a freezer at $-80{ }^{\circ} \mathrm{C}$. All participants were requested to provide basic information about their clinical characteristics, and complete the International Prostate Symptom Score (IPSS) questionnaire which includes a total of 8 questions: 7 symptom questions (score $0-5$ points) and one quality of life due to urinary symptoms question (QOL, score 0-6 points) [20]. We also checked the laboratory data for all participants, including fasting blood glucose, hemoglobin A1c (HbA1c), cholesterol, 
triglyceride, low-density lipoproteins (LDL), high-density lipoproteins (HDL), glutamic oxaloacetic transaminase (GOT), glutamic pyruvic transaminase (GPT), blood urea nitrogen (BUN), creatinine, prostate specific antigen (PSA), free PSA, testosterone, and insulin. The size of the prostate of $\mathrm{BPH}$ patients was calculated using sonography. The clinical characteristics of the patients are shown in Table 1. For every participant, $10-20 \mathrm{ml}$ urine sample were centrifuged at $14000 \mathrm{rpm}$ for $15 \mathrm{~min}$ to collect the microbe. After centrifugation, supernatant discard and the pellet was resuspended with 500ul ST1 buffer (CatchGeneTM Stool DNA Kit content) for the cell lysis step according to the manuscript of CatchGene stool DNA extraction kit. Each step of the procedure, including the extraction of the genomic DNA; amplification by PCR; PCR product quantification, mixing, and purification; DNA library preparation; and sequencing, was performed with quality control (QC) measures. DNA was extracted from the precipitate using the CatchGeneTM Stool DNA Kit (CatchGene, Taiwan). For

Table 1 Comparisons of demographic, clinical characteristics and bacteria alpha diversity between BPH group and normal control group

\begin{tabular}{|c|c|c|c|}
\hline & $\mathrm{BPH}(\mathrm{n}=77)$ & Control $(n=30)$ & $P$ value \\
\hline \multicolumn{4}{|l|}{ Demographic } \\
\hline Age & $69.44 \pm 8.23$ & $61.97 \pm 8.32$ & $<0.001^{\mathrm{a}}$ \\
\hline Body mass index & $24.17 \pm 3.33$ & $24.55 \pm 2.87$ & 0.616 \\
\hline \multicolumn{4}{|l|}{ Clinical Characteristics } \\
\hline Fasting blood glucose & $110.89 \pm 17.05$ & $114.48 \pm 26.77$ & 0.436 \\
\hline $\begin{array}{l}\text { Prostate specific antigen } \\
\text { (PSA) }\end{array}$ & $3.02 \pm 3.21$ & $2.37 \pm 3.94$ & 0.406 \\
\hline Free PSA & $0.60 \pm 0.11$ & $0.69 \pm 1.25$ & 0.613 \\
\hline Hemoglobin A1c (\%) & $5.81 \pm 0.67$ & $5.88 \pm 0.53$ & 0.660 \\
\hline Creatinine & $0.98 \pm 0.22$ & $0.93 \pm 0.18$ & 0.393 \\
\hline BUN & $15.44 \pm 4.94$ & $13.22 \pm 2.93$ & 0.036 \\
\hline $\mathrm{HDL}$ & $53.93 \pm 24.43$ & $47.91 \pm 11.76$ & 0.239 \\
\hline Cholesterol & $181.51 \pm 42.61$ & $182.28 \pm 28.15$ & 0.933 \\
\hline LDL & $110.61 \pm 33.82$ & $116.75 \pm 26.46$ & 0.409 \\
\hline Testosterone & $551.68 \pm 246.50$ & $540.75 \pm 158.96$ & 0.836 \\
\hline GOT & $25.96 \pm 7.63$ & $26.50 \pm 7.79$ & 0.804 \\
\hline GPT & $26.133 \pm 12.86$ & $30.56 \pm 20.82$ & 0.210 \\
\hline TG & $108.68 \pm 66.63$ & $102.52 \pm 44.11$ & 0.667 \\
\hline IPSS & $6.25 \pm 4.19$ & $2.16 \pm 1.37$ & $<0.001^{\mathrm{a}}$ \\
\hline Quality of life scores & $2.09 \pm 0.68$ & $1.2 \pm 0.41$ & $<0.001^{\mathrm{a}}$ \\
\hline \multicolumn{4}{|c|}{ Parameter of Bacterial Alpha Diversity } \\
\hline Observed species & $256.17 \pm 65.29$ & $254.70 \pm 51.58$ & 0.912 \\
\hline Chao1 & $284.40 \pm 65.63$ & $288.21 \pm 63.05$ & 0.786 \\
\hline ACE index & $277.65 \pm 64.78$ & $280.21 \pm 57.85$ & 0.850 \\
\hline Shannon index & $5.81 \pm 1.04$ & $5.59 \pm 0.90$ & 0.314 \\
\hline Simpson index & $0.93 \pm 0.08$ & $0.92 \pm 0.06$ & 0.697 \\
\hline
\end{tabular}

a IPSS: International Prostate Symptom Score the negative control, double-distilled water $\left(\mathrm{ddH}_{2} \mathrm{O}\right)$ was used as the sample for the extraction process, and the eluate was further used as the negative control sample for the PCR amplification step. Specific primers (319 F: 5'-CCTACGGGNGGCWGCAG-3', 806 R: 5'-GACTAC HVGGGTATCTAATCC-3') were used to perform the PCR amplification of $16 \mathrm{~S}$ rDNA over the V3-V4 regions according to the $16 \mathrm{~S}$ Metagenomic Sequencing Library Preparation procedure (Illumina). The indexed PCR product quality was assessed using the Qubit 4.0 fluorometer (Thermo Scientific) and Qsep100 ${ }^{\mathrm{TM}}$ system. An equal amount of the indexed PCR product was mixed to generate the sequencing library. Finally, the library was sequenced on the MiSeq platform (Illumina), and pairedend 300-bp reads were generated. The raw data were merged and filtered to obtain clean data. Operational taxonomic unit (OTU) clusters were obtained from the data.

\section{Statistical analysis}

We used Student's $t$-test for continuous variables to evaluate the differences in the clinical characteristics between the participants of the $\mathrm{BPH}$ and control groups. For urine microbiota analysis, we tested the significance of community composition and structural differences between the groups using ANOSIM. Welch's $t$-test was used to identify the significant differences in species between the groups $(P<0.05)$ at various taxon levels, including the phylum, class, order, family, genus, and species. Pearson's correlation analysis was performed to calculate the relationship between the clinical characteristics of the participants and the microbiota species in the urine samples. SPSS (Statistical Product and Service Solutions, version 22) software was used to analyze the data, and $P$ values $<0.05$ were considered statistically significant.

\section{Bioinformatic analysis}

By using the FLASH (Fast Length Adjustment of Short Reads, v.1.2.11) software, we assembled the $300 \mathrm{bp}$ paired-end raw reads derived from $16 \mathrm{~S}$ rDNA amplicon sequencing [21]. For each representative sequence, the RDP (Ribosomal Database Project, v.2.2) classifier algorithm was employed to annotate taxonomy classification based on the information retrieved from the Silva Database v.132 [22]. Subsequent analysis of alpha and beta diversities was performed using the normalized data. Alpha diversity was indicative of the species complexity within individual urine samples based on six different criteria output from the QIIME (Quantitative Insights Into Microbial Ecology) pipeline, including observed OTUs, and Chao-1, Shannon, Simpson, ACE (Abundance-based Coverage Estimator), and Good's coverage indices [23]. Beta diversity analysis was used to evaluate 
the differences among the samples in terms of species complexity. Two beta diversity parameters, the weighted and unweighted UniFrac [24], were calculated using the QIIME pipeline. Principal coordinate analysis (PCoA) was performed to acquire principal coordinates for the visualization of sophisticated and multidimensional data. PCoA analysis was conducted using the WGCNA (Weighted Correlation Network Analysis), stat, and ggplot2 packages in $\mathrm{R}$ software (v.2.15.3). Non-metric multidimensional scaling (NMDS) analysis was performed to fit the nonlinear model in ecological datasets by using the vegan package in $\mathrm{R}$ software (v.2.15.3) $[25,26]$. The significance of all species among groups at various taxonomic levels was detected using differential abundance analysis with a zero-inflated Gaussian (ZIG) log-normal model as implemented in the "fitFeatureModel" function of the metagenomeSeq package of the Bioconductor software [27]. Statistically significant biomarkers were identified using LEfSe (linear discriminant analysis effect size) method [28]. Taxa with an LDA (linear discriminant analysis) score $(\log 10)>4$ were considered significant [27].

\section{Results}

\section{Demographic and clinical characteristics} of the participants

We enrolled $77 \mathrm{BPH}$ patients and 30 control participants into two groups in our microbiota analysis. All urine samples were collected with sterile tubes and provided adequate sequencing reads, named as UBPH and UN. This can be verified from the Rarefaction curve, which became a smooth reflection of reasonable species richness in Fig. 1. Detailed comparisons between the BPH and control groups are shown in Table 1. IPSS, QOL and age were significantly different between the participants of the two groups. More severity of LUTS, poor quality of life, and older age were seen in the patients of the $\mathrm{BPH}$ group than in the participants of the control group (IPSS for BPH group: $6.25 \pm 4.19$, IPSS for control group: $2.16 \pm 1.37$; QOL score for BPH group: $2.09 \pm 0.68$, QOL score for control group: $1.2 \pm 0.41$; age for $\mathrm{BPH}$ group: $69.44 \pm 8.23$, age for control group: $61.97 \pm 8.32$ ). No significant differences were observed in other clinical characteristics of the participants of both groups.

\section{Bioinformatic analysis}

From the Venn diagram, 2617 OTUs were identified in the samples of the BPH group and 1820 OTUs were identified in the samples of the control group. The groups had 1381 OTUs in common. Comparisons of alpha diversity between the samples of the $\mathrm{BPH}$ and control groups are also seen in Table 1 . There were no significant differences between the BPH and control groups in terms

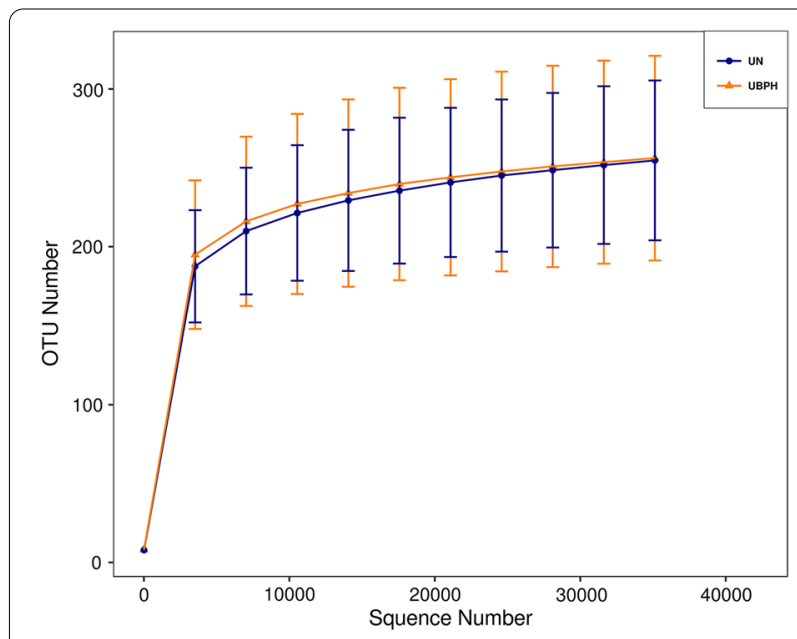

Fig. 1 Rarefaction curve: The curve becomes flatter which means almost samples have been taken

of observed species ( $256.17 \pm 65.29$ vs. $254.70 \pm 51.58)$, Chao-1 index (284.40 \pm 65.63 vs. $288.21 \pm 63.05)$, ACE index $\quad(277.65 \pm 64.78$ vs. $280.21 \pm 57.85)$, Shannon index $(5.81 \pm 1.04$ vs. $5.59 \pm 0.90)$ and Simpson index $(0.93 \pm 0.08$ vs. $0.92 \pm 0.06)$. As shown in Fig. 2 through $\mathrm{PCoA}$, we found urinary microbiota in $\mathrm{BPH}$ patients was distinct from that of control group. Statistically differences were observed after t-test analysis distance metrics $(P<0.001, P<0.001$ for weighted UniFrac, unweighted UniFrac). A similar distribution can also be found in the non-metric multidimensional scaling (NMDS) shown in Fig. 3. We compared the differences between the samples in the form of a heat tree. The circular heat tree represents the richness of different taxonomy levels by node size, edge thickness, and color (Fig. 4a, b).

Based on the results of species annotation, we identified the 10 most abundant species at genus level to form the distribution histogram of relative abundance in Fig. 5. The top ten bacterial genera in descending order of abundance in the urine samples were Sphingomonas, Bacteroides, Lactobacillus, Streptococcus, Alcaligenes, Prevotella, Ruminococcaceae UCG-014, Escherichia_Shigella, Akkermansia, and Parabacteroides. From the results of ANOSIM statistical analysis in Table 2, a significant difference was observed between the BPH and control groups $(P=0.01)$. STAMP (Statistical analysis of metagenomic profiles) software was used to perform Welch's $t$-test and discover the significant difference between the groups at the genus level, as shown in Fig. 6. The bar graph reveals that the 15 genera which were significantly expressed in the samples of the BPH group, are Lactobacillus, Staphylococcus, Bacillus, Faecalibacterium, 


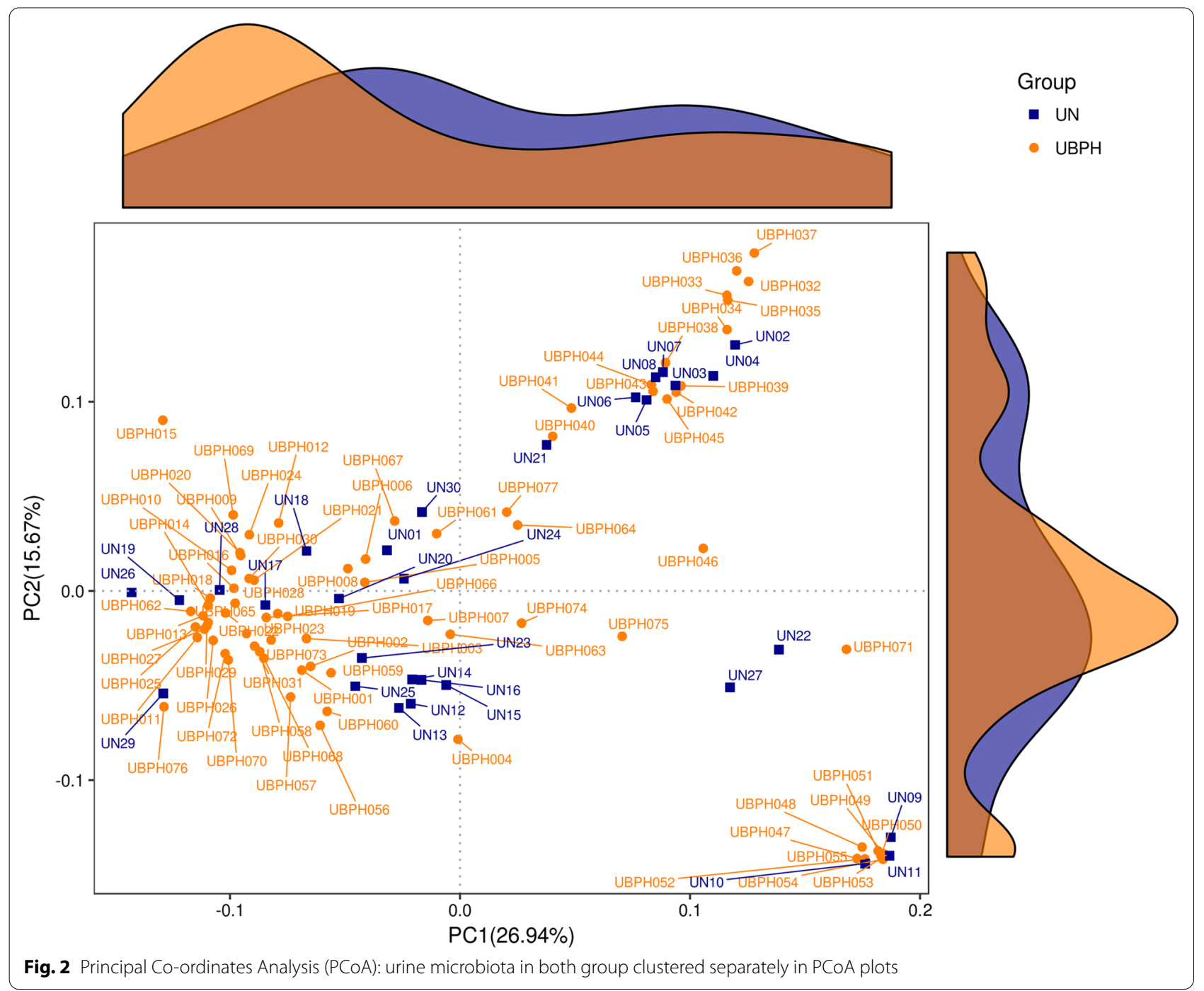

Listeria, Enhydrobacter, Pseudomonas, Neisseria, Phascolarctobacterium, Dolosigranulum, Haemophilus, [Ruminococcus]torques, Bamesiella, Finegoldia, and Prevotellaceae NK3B31 group.

\section{Correlation analysis}

In Fig. 7, Spearman's correlation analysis revealed that urine samples with Haemophilus, Staphylococcus, Dolosigranulum, Listeria, Phascolarctobacterium, Enhydrobacter, Bacillus, [Ruminococcus]torques, Faecalibacterium, and Finegoldia presented high IPSS, and severe storage and voiding symptoms $(P<0.05)$. The urine samples with a higher abundance of Haemophilus showed higher PSA levels while the presence of Lactobacillus in urine samples $(P<0.05)$ was positively associated with high HbA1c and glucose levels.

\section{Discussion}

Since the importance of microbiota was established, numerous studies have addressed the impact of microbiota and the balance between host and resident microbiota on the human body [11, 29]. In addition to gut microbiota, urine microbiota also impacts urinary diseases, but is poorly understood. In this study, we describe the urinary microbiota in males with and without $\mathrm{BPH}$, and establish the association between abundance of species and clinical characteristics of participants, using $16 \mathrm{~S}$ rDNA amplicon sequencing. To our knowledge, few studies have discussed the detailed impact of urinary microbiota in BPH patients. It was incorrectly considered that urine is sterile, primarily on the basis of culture-dependent methods. However, microbes exist in urine of adult men and women even without clinical infection $[10,16]$. 


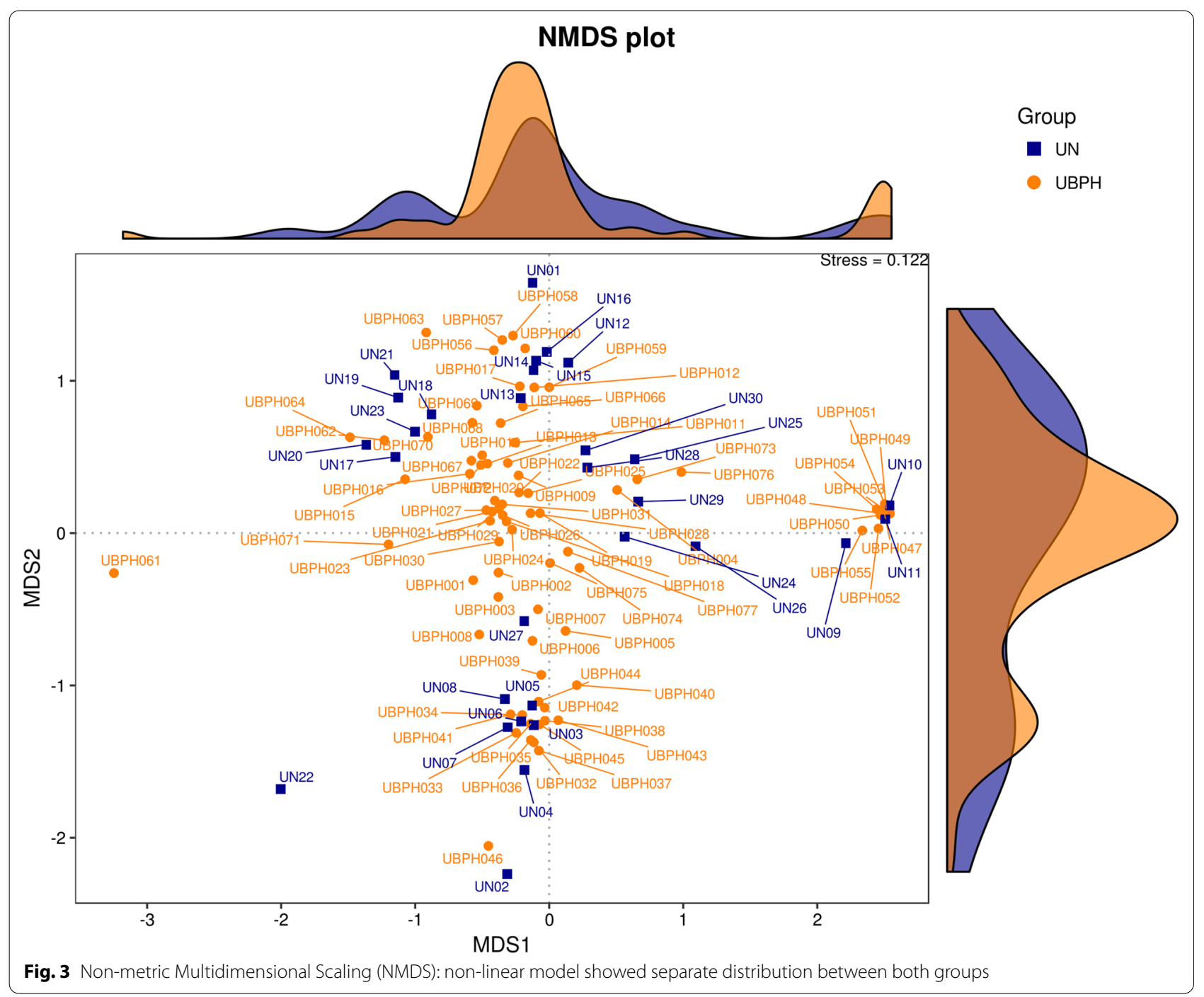

In a study by Lewis et al. [9], 16S rRNA sequencing on mid-stream voided urine samples revealed that the number and diversity of microbiota change with age in healthy, asymptomatic individuals of both genders. It is assumed that these changes may be associated with the occurrence of BPH with aging, in men [30]. Although both BPH and LUTS are highly prevalent in men and increase in prevalence with age, only $25-50 \%$ of patients with BPH suffer from significant LUTS, while only half to two-thirds of patients with LUTS showed bladder outlet obstruction (BOO) on urodynamic testing [31]. In addition, the difference in individual response suggests that the pathophysiology of $\mathrm{BPH}$ is heterogeneous and poorly understood. The diversity within $\mathrm{BPH}$ participants also can be demonstrated in our results. The causes of $\mathrm{BPH} / \mathrm{LUTS}$ are multifactorial, and inflammation is one of the important factors that was proposed on observation of chronic inflammation coexisting with histologic changes in resected prostate specimen in $\mathrm{BPH}$ [32]. Prostate inflammation may be triggered by bacterial infection, followed by the secretion of cytokines, chemokines, and growth factors including CD4, CD8, CD45, CD68, C-reactive protein, tumor necrosis factor, interleukin-6, and others [33-35]. Due to the above hypothesis, a link between $\mathrm{BPH}$ and microbiota was considered and a target for its diagnosis and treatment was set.

LUTS is composed of voiding and storage symptoms, which were mainly evaluated by IPSS. Clinically, we can stratify mild to severe symptoms with a symptom score. Bajic et al. [20] observed a distinct presence of microbiota in severe symptomatic patients who needed surgical therapy, and in minimally symptomatic controls. Compared to the study by Bajic et al., our results detected statistically significant differences in the relative abundance of specific microbes in the urine samples of $\mathrm{BPH}$ patients compared to the samples of the 


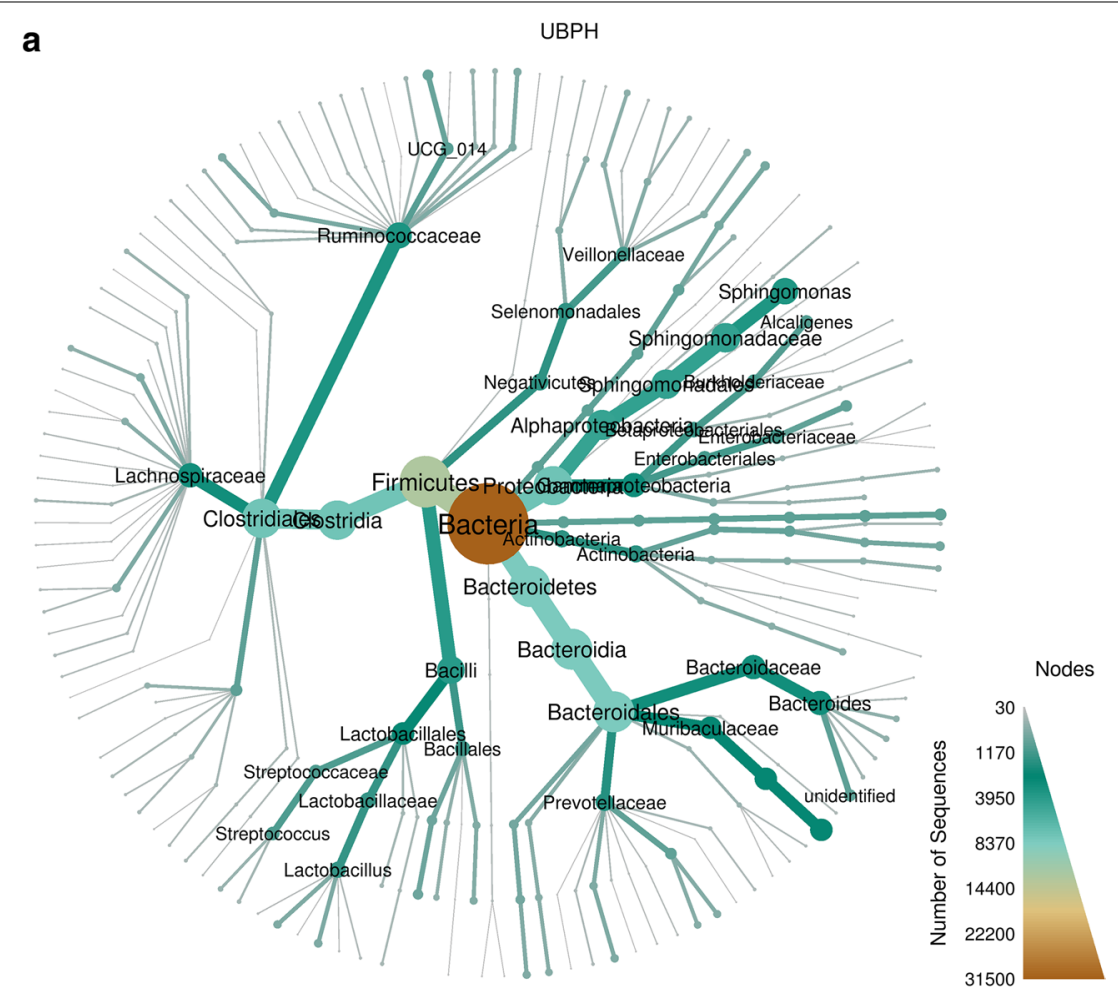

b

UN

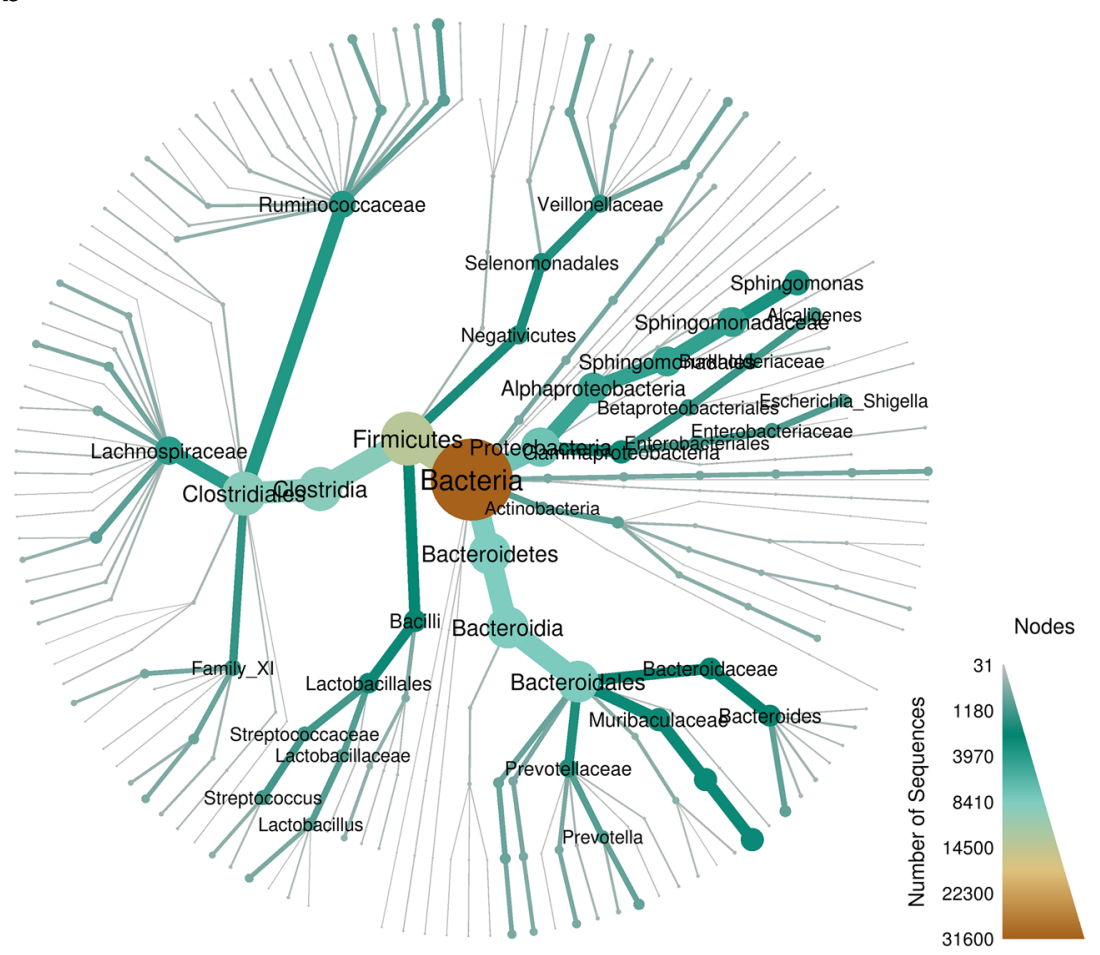

Fig. 4 Heat tree: it presents richness of different taxonomy level by node size, edge thickness and color in (a): BPH group and (b): normal control group 


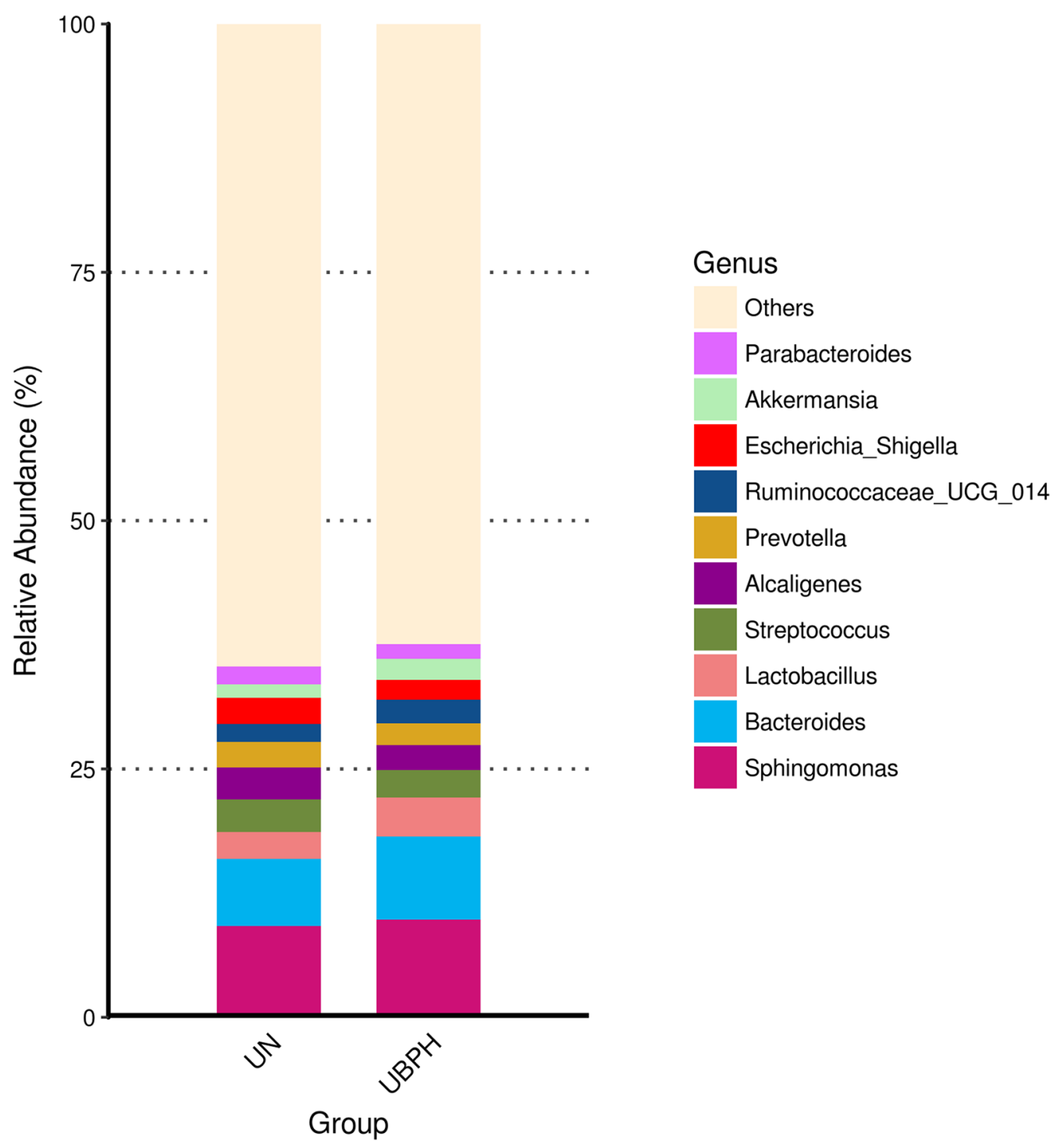

Fig. 5 Top 10 abundant species comparing BPH and control group at the genus level

Table 2 Anosim statistic analysis: comparing the difference of the composition of microbiota between BPH group and normal control group

\begin{tabular}{llc}
\hline Group & R-value & P-value \\
\hline UN-UBPH & 0.115 & 0.01 \\
\hline
\end{tabular}

control group. Further evaluation of the urine microbiota elucidates association of some microbes with degrees of LUTS as well as with the voiding and storage symptoms. Significant differences in microbes were also present in this subset of symptoms. Nevertheless, it is hypothesized that dysbiosis of the urine microbiota may play an important role in the progression of $\mathrm{BPH}$ and LUTS. Within pathogens that over-represented in urine samples of BPH patients, Haemophilus, Staphylococcus, Dolosigranulum, Listeria, Phascolarctobacterium, Enhydrobacter, Bacillus, [Ruminococcus]torques, Faecalibacterium, and Finegoldia are associated with more severe LUTS.

Haemophilus spp. is reported as a rare urinary tract pathogen but needs to be investigated in patients with urinary tract abnormalities such as urolithiasis [36]. A previous study demonstrated that Faecalibacterium was more abundant in patients with diabetes mellitus (DM) and hyperlipidemia, compared to patients with only DM, as seen in the study by Fengping Liu et al. 


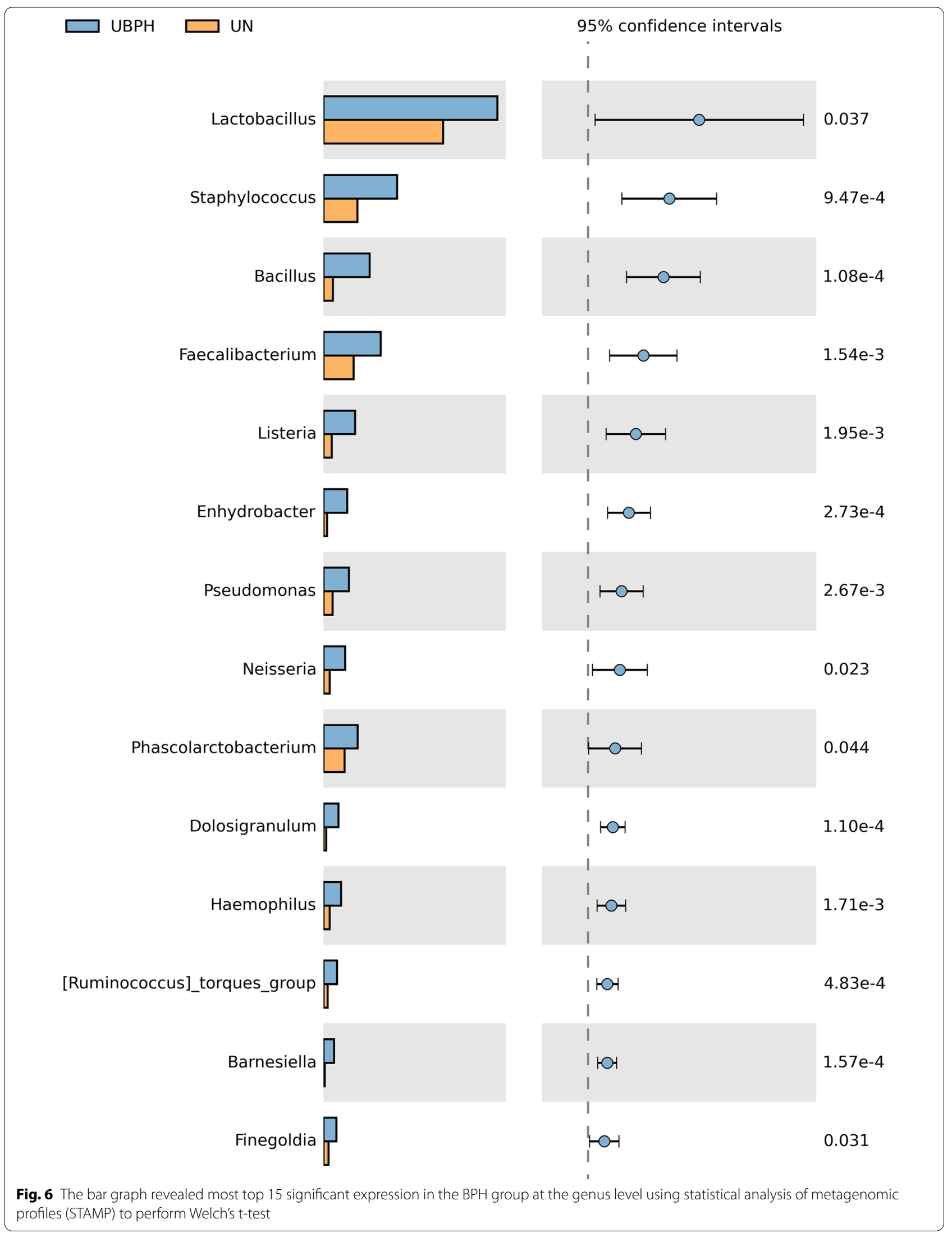




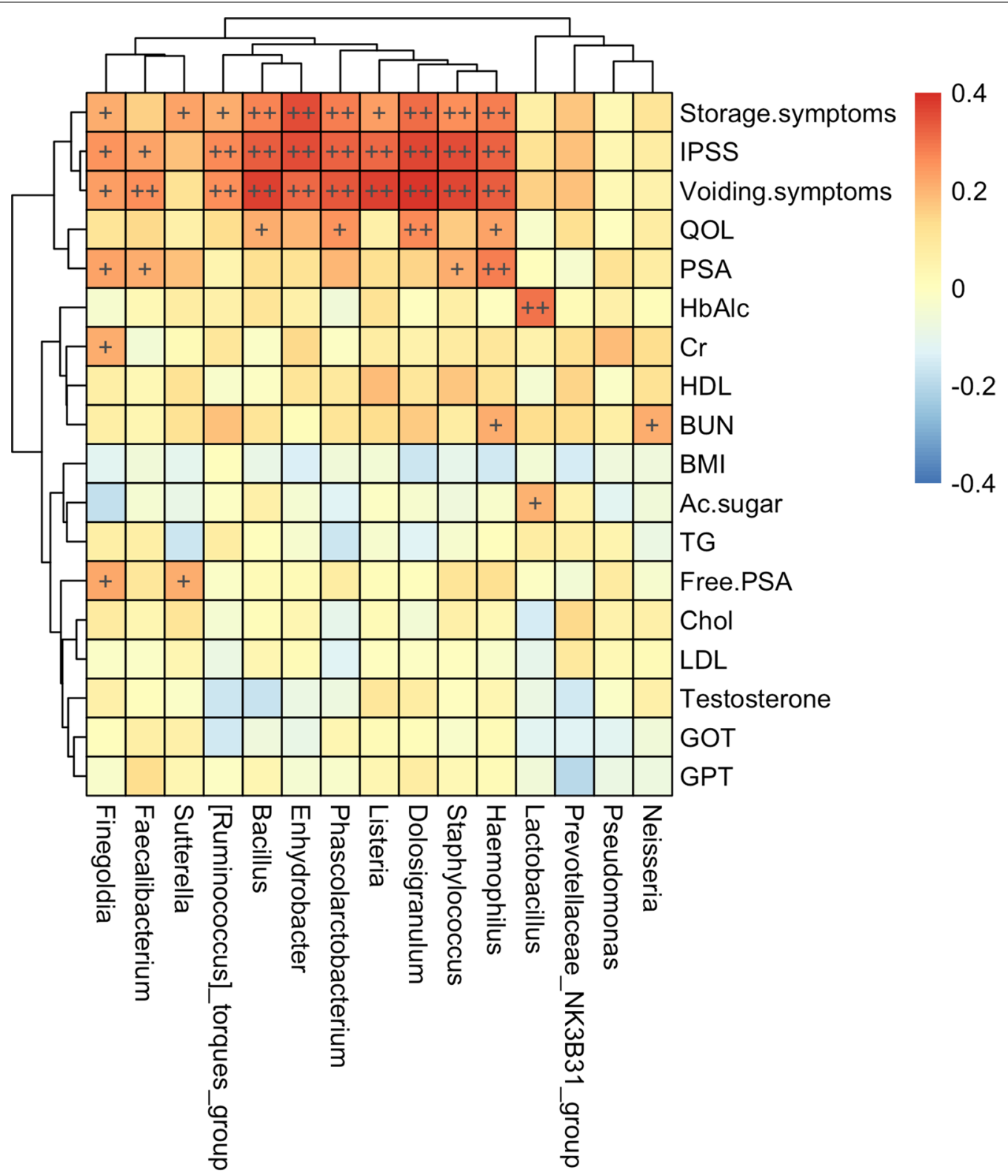

Fig. 7 Spearman correlation analysis: identify significant correlation between specific microbes and clinical characteristics

They also demonstrated that Faecalibacterium is associated with incidence of UUI. In addition, Bacillus is also common in patients with DM, hypertension and hyperlipidemia [37]. After culture-independent $16 \mathrm{~S}$ rDNA gene sequencing, Finegoldia was identified in urine samples from patients with severe preoperative urinary symptoms, undergoing surgery for pelvic organ prolapse and stress incontinence [38]. The presence of Lactobacillus was positively associated with $\mathrm{HbA1c}$ and glucose levels in our study as well as in the study by Jiawei Chen et al. Their results suggested that changes in the microbial community, specifically in Lactobacillus, may be an impact factor in LUTS [39]. According to previous studies, microbes can be identified in culturenegative urine.

In order to decrease the possibility of urine contamination, we collected mid-stream voided urine samples with sterile containers. Furthermore, voided urine is less easily contaminated with microbes in males than in females. Although collecting urine by sterile catheterization was an alternative method for our study, it was considered invasive, uncomfortable and not clinically feasible. 
Different urine collection methods show different urine microbiota. This may be due to differences between the microbiome of the urethra and the bladder [40]. According to this hypothesis, results of the urine microbiota in our study may represent a mixture of bacteria from the urethra and the bladder. Because antibiotic treatment can impact the composition of urine microbiota and the influence may persist for at least two months [41], we excluded participants who had taken antibiotics within the last two months.

Our study has some limitations. First, it is a crosssectional study in which the cause-effect relationship is difficult to define between clinical characteristics and bioinformatics indicators. Therefore, animal experimentation is needed to clarify the role of specific microbes in the development of BPH and LUTS. Second, we only analyzed urine microbiota from voided urine; further work would include catheterized urine collection to identify the different microbes residing in the bladder and urethra. Furthermore, we did not analyze the relationship between the medications for $\mathrm{BPH}$ and urine microbiota. Thus, prospective studies need to clarify if different strategies will disturb the environment of the microbiota. Finally, another weakness is the lack of replicates, other cohort to verify the composition of urinary microbiota in $\mathrm{BPH}$ patients is justified.

\section{Conclusion}

We have outlined the bacterial microbiota in the urine of $\mathrm{BPH}$ patients is different from that seen in control participants but some may be indistinguishable from control group. We also discovered that urine microbiota is significantly associated with the severity of LUTS, even in a subset of symptoms, and confirmed what Bajic et al. have demonstrated. The results suggest that some specific microbes may have important roles in the development and progression of BPH. Further research is needed to target these microbes for analyzing the pathobiology of BPH and improving LUTS.

\section{Acknowledgements \\ This study was supported by grants from Kaohsiung Municipal Ta-Tung Hos- pital (Kmtth-106M-001), from the Ministry of Science and Technology (MOST 109-2314-B-037-089) and supported partially by Kaohsiung Medical University Research Center Grant (KMU-TC108A02-2, KMU-TC108A02-4). The authors would like to thank Dr. Yu-Lun Kuo at BIOTOOLS Co., Ltd in Taiwan for kindly supporting analysis of NGS data.}

\section{Authors' contributions}

HYL: Study design, collecting data, drafted manuscript. JWW: Study design, collecting data. YSJ: Study design. CCL: Collecting data. CJL: Bioinformatics and taxonomic analysis. SYC: Revision of the manuscript. HCY: Statistic analysis. KSC: Collecting data. WJW: Study design, collecting data. DCW: Study design, collecting data. All authors read and approved the final manuscript.

\section{Funding}

Not applicable.
Availability of data and materials Not applicable.

\section{Declarations}

\section{Ethics approval and consent to participate}

This article is approved by the Kaohsiung Medical University Hospital Institutional Review Board (KMUHIRB-F(I)-20190013). Informed consent was obtained from all participants.

\section{Consent for publication}

Not applicable.

\section{Competing interests}

The authors declare no competing financial interests.

\section{Author details}

${ }^{1}$ Department of Urology, Kaohsiung Municipal Ta-Tung Hospital, Kaohsiung, Taiwan. ${ }^{2}$ Graduate Institute of Clinical Medicine, College of Medicine, Kaohsiung Medical University, Kaohsiung, Taiwan. ${ }^{3}$ Department of Urology, Kaohsiung Medical University Hospital, Kaohsiung, Taiwan. ${ }^{4}$ Department of Urology, School of Medicine, College of Medicine, Kaohsiung Medical University, Kaohsiung, Taiwan. ${ }^{5}$ Division of Gastroenterology, Department of Internal Medicine, Kaohsiung Medical University Hospital, Kaohsiung Medical University, Kaohsiung, Taiwan. ${ }^{6}$ Regenetative Medicine and Cell Therapy Research Center, Kaohsiung Medical University, No.100, Tzyou 1st Rd., Sanmin Dist., Kaohsiung 80756, Taiwan. ${ }^{7}$ Department of Urology, Seoul National University Hospital, Seoul, Korea. ${ }^{8}$ Department of Medicine, Faculty of Medicine, College of Medicine, Kaohsiung Medical University, Kaohsiung, Taiwan.

Received: 1 October 2020 Accepted: 7 April 2021

Published online: 15 April 2021

References

1. Wei JT, Calhoun E, Jacobsen SJ. Urologic diseases in America project: Benign prostatic hyperplasia. J Urol. 2005;173:1256-61.

2. Lee SW, Chan EM, Lai YK. The global burden of lower urinary tract symptoms suggestive of benign prostatic hyperplasia: a systematic review and meta-analysis. Sci Rep. 2017;7:7984.

3. Lokeshwar SD, Harper BT, Webb E, Jordan A, Dykes TA, Neal DE, et al. Epidemiology and treatment modalities for the management of benign prostatic hyperplasia. Transl Androl Urol. 2019;8(5):529-39.

4. Patel ND, Kellogg JK. Epidemiology and etiology of benign prostatic hyperplasia and bladder outlet obstruction. Indian J Urol. 2014;30(2):170-6.

5. Anim JT, Udo C, John B. Characterisation of inflammatory cells in benign prostatic hyperplasia. Acta Histochem. 1998;100:439-49.

6. Nickel JC. Inflammation and benign prostatic hyperplasia. Urol Clin North Am. 2008;35:109-15.

7. Sutcliffe S, Giovannucci E, De Marzo AM, Willett WC, Platz EA. Sexually transmitted infections, prostatitis, ejaculation frequency, and the odds of lower urinary tract symptoms. Am J Epidemiol. 2005;162:898-906.

8. Alegre M-L, Mannon RB, Mannon PJ. The Microbiota, the Immune System and the Allograft. Am J Transplant. 2014;14(6):1236-48.

9. Lewis DA, Brown R, Williams J, White P, Jacobson SK, Marchesi JR, et al. The human urinary microbiome; bacterial DNA in voided urine of asymptomatic adults. Front Cell Infect Microbiol. 2013;3:41.

10. Wolfe AJ, Toh E, Shibata N, Rong R, Kenton K, Fitzgerald M, et al. Evidence of uncultivated bacteria in the adult female bladder. J Clin Microbiol. 2012;50:1376-83.

11. Nelson DE, Van Der Pol B, Dong Q, Revanna KV, Fan B, Easwaran S, et al. Characteristic male urine microbiomes associate with asymptomatic sexually transmitted infection. PLoS ONE. 2010;5(11):e14116.

12. Brubaker L, Nager CW, Richter HE, Visco A, Nygaard I, Barber MD, et al. Urinary bacteria in adult women with urgency urinary incontinence. Int Urogynecol J. 2014;25(9):1179-84. 
13. Pearce MM, Hilt EE, Rosenfeld AB, Zilliox MJ, Thomas-White K, Fok C, et al. The Female Urinary Microbiome: a Comparison of Women with and without Urgency Urinary Incontinence. Bio. 2014;5(4):01283-314.

14. Pearce MM, Zilliox MJ, Thomaswhite KJ, Richter HE, Nager CW, Visco AG, et al. The female urinary microbiota in urgency urinary incontinence. Am J Obstet Gynecol. 2015;213(3):347.e1-347.e11.

15. Khasriya R, Sathiananthamoorthy S, Ismail S, Kelsey M, Wilson M, Rohn $J$, Malone-Lee J. Spectrum of bacterial colonization associated with urothelial cells from patients with chronic lower urinary tract symptoms. J Clin Microbiol. 2013;51(7):2054-62.

16. Hilt EE, McKinley K, Pearce MM, Rosenfeld AB, Zilliox MJ, Mueller ER, et al. Urine is not sterile: use of enhanced urine culture techniques to detect resident bacterial flora in the adult female bladder. J Clin Microbiol. 2014;52(3):871-6.

17. Lloyd-Price J, Abu-Ali G, Huttenhower C. The healthy human microbiome. Genome Med. 2016;8(1):51.

18. Heytens S, De Sutter A, Coorevits L, Cools P, Boelens J, Van Simaey L, et al. Women with symptoms of a urinary tract infection but a negative urine culture: PCR-based quantification of Escherichia coli suggests infection in most cases. Clin Microbiol Infect. 2017;23(9):647-52.

19. Yu H, Meng H, Zhou F, Ni X, Shen S, Das UN. Urinary microbiota in patients with prostate cancer and benign prostatic hyperplasia. Arch Med Sci. 2015;11(2):385-94.

20. Bajic P, van Kuiken ME, Burge BK, Kirshenbaum EJ, Joyce CJ, Wolfe AJ, et al. Male bladder microbiome relates to lower urinary tract symptoms. Eur Urol Focus. 2020;6(2):376-82.

21. Magoc T, Salzberg SL. FLASH: fast length adjustment of short reads to improve genome assemblies. Bioinformatics. 2011;27(21):2957-63.

22. Quast C, Pruesse E, Yilmaz P, Gerken J, Schweer T, Yarza P, et al. The SILVA ribosomal RNA gene database project: improved data processing and web-based tools. Nucleic Acids Res. 2013:41(Database issue):D590-6.

23. Whittaker RH. Evolution and measurement of species diversity. Taxon. 1972:21:213-51.

24. Lozupone C, Lladser ME, Knights D, Stombaugh J, Knight R. UniFrac: an effective distance metric for microbial community comparison. ISME J. 2011;5(2):169-72.

25. Jiang $X-T$, Peng $X$, Deng G-H, Hua-Fang Sheng Yu, Wang H-W, et al. Illumina sequencing of $16 \mathrm{~S}$ rRNA tag revealed spatial variations of bacterial communities in a mangrove wetland. Microb Ecol. 2013;66(1):96-104.

26. Rivas MN, Burton OT, Wise P, Zhang YQ, Hobson SA, Lloret MG, et al. A microbiota signature associated with experimental food allergy promotes allergic sensitization and anaphylaxis. J Allergy Clin Immunol. 2013;131(1):201-12.

27. Paulson JN, Stine OC, Bravo HC, Pop M. Differential abundance analysis for microbial marker-gene surveys. Nat Methods. 2013;10(12):1200-2.

28. Segata N, Izard J, Waldron L, Gevers D, Miropolsky L, Garrett WS, et al. Metagenomic biomarker discovery and explanation. Genome Biol. 2011;12(6):R60.
29. Ostaff MJ, Stange EF, Wehkamp J. Antimicrobial peptides and gut microbiota in homeostasis and pathology. EMBO Mol Med. 2013;5:1465-83.

30. Bajic P, Dornbier RA, Doshi CP, Wolfe AJ, Farooq AV, Bresler L. Implications of the genitourinary microbiota in prostatic disease. Curr Urol Rep. 2019;20(7):34.

31. Chen JL, Jiang YH, Lee CL, Kuo HC. Precision medicine in the diagnosis and treatment of male lower urinary tract symptoms suggestive of benign prostatic hyperplasia. Ci Ji Yi Xue Za Zhi. 2019;32(1):5-13.

32. Lloyd GL, Marks JM, Ricke WA. Benign prostatic hyperplasia and lower urinary tract symptoms: what is the role and significance of inflammation? Curr Urol Rep. 2019;20(9):54.

33. Torkko KC, Wilson RS, Smith EE, Kusek JW, van Bokhoven A, Lucia MS. Prostate biopsy markers of inflammation are associated with risk of clinical progression of benign prostatic hyperplasia: findings from the MTOPS study. J Urol. 2015;194:454-61.

34. Schenk JM, Kristal AR, Neuhouser ML, Tangen CM, White E, Lin DW, et al. Biomarkers of systemic inflammation and risk of incident, symptomatic benign prostatic hyperplasia: results from the prostate cancer prevention trial. Am J Epidemiol. 2010;171:571-82.

35. Madersbacher S, Sampson N, Culig Z. Pathophysiology of benign prostatic hyperplasia and benign prostatic enlargement: a mini-review. Gerontology. 2019;65(5):458-64.

36. Stærk M, Tolouee SA, Christensen JJ. Nontypable haemophilus influenzae septicemia and urinary tract infection associated with renal stone disease. Open Microbiol J. 2018;12:243-7.

37. Liu F, Ling Z, Xiao Y, Yang Q, Wang B, Zheng L, et al. Alterations of urinary microbiota in type 2 diabetes mellitus with hypertension and/or hyperlipidemia. Front Physiol. 2017;8:126.

38. Fok CS, Gao X, Lin H, Thomas-White KJ, Mueller ER, Wolfe AJ, et al. Urinary symptoms are associated with certain urinary microbes in urogynecologic surgical patients. Int Urogynecol J. 2018;29(12):1765-71.

39. Chen J, Zhao J, Cao Y, Zhang G, Chen Y, Zhong J, et al. Relationship between alterations of urinary microbiota and cultured negative lower urinary tract symptoms in female type 2 diabetes patients. BMC Urol. 2019:19(1):78.

40. Pohl HG, Groah SL, Pérez-Losada M, Ljungberg I, Sprague BM, Chandal N, et al. The urine microbiome of healthy men and women differs by urine collection method. Int Neurourol J. 2020;24(1):41-51.

41. Dethlefsen L, Relman DA. Incomplete recovery and individualized responses of the human distal gut microbiota to repeated antibiotic perturbation. Proc Natl Acad Sci USA. 2011;108(1):4554-61.

\section{Publisher's Note}

Springer Nature remains neutral with regard to jurisdictional claims in published maps and institutional affiliations.
Ready to submit your research? Choose BMC and benefit from:

- fast, convenient online submission

- thorough peer review by experienced researchers in your field

- rapid publication on acceptance

- support for research data, including large and complex data types

- gold Open Access which fosters wider collaboration and increased citations

- maximum visibility for your research: over $100 \mathrm{M}$ website views per year

At BMC, research is always in progress.

Learn more biomedcentral.com/submissions 